\section{GLYCOGEN BREAKDOWN}

Enzyme Ensemble

from our Molecular Biology Correspondent

ChargafF's deathless adage that molecular biology is merely biochemistry practised without a licence may seem to find support in comparisons with work such as that of Fischer, Krebs and others on the system of enzymes responsible for the breakdown of glycogen. What at best characterizes the thinking of the fully licensed practitioner, and is so striking a feature of this work, is the manner in which the objectives and results are related to the broad biological design, and are brought ever closer to the situation in the cell. The latest successes in this direction are described in three massive articles from Fischer's laboratory on the interrelation between the enzymes of glycogen breakdown in newly discovered subcellular particles prepared from muscle.

The breakdown of glycogen, in accordance with instantaneous energy requirements, is controlled by the activity of glycogen phosphorylase. This enzyme exists in two states: phosphorylase $b$, at least in vivo at high concentration, is a form of low activity, which is activated to a modest degree by AMP. Under the action of phosphorylase kinase it is phosphorylated at one serine residue in each of the four subunits, to give the highly active phosphorylase $a$. The kinase is itself activated by calcium ions, with the additional complication that a calcium-dependent protease, present in the tissue, also causes activation, perhaps adventitious, of the kinase by limited proteolysis. To complete the cycle, the $b$ form is regenerated from the $a$ by the action of a phosphatase, which is liable to inhibition by nucleotides.

Meyer et al. (J. Biol. Chem., 245, $6642 ; 1970)$ now show that all these enzymes coexist in glycogen particles, 200-300 $\AA$ in diameter, which can be prepared in several ways from muscle. The preparations also contain much larger vesicles, apparently derived from the sarcoplasmic reticulum. These can be separated from each other by sedimentation or gel filtration, and the phosphorylase and its satellite enzymes appear in the smaller glycogen particles, whereas the vesicle fraction contains highly active calcium pump ATPase.

In the second article (Heilmeyer et al., ibid., 6649) the mechanism of control of phosphorylase activity in these particles is examined. The phosphorylase in situ is entirely in the $b$ form, and thus essentially inactive. The phosphorylase kinase is also dormant, but the phosphatase is active, so that no conversion to phosphorylase $a$ is possible. When now magnesium ATP and calcium are added, there is a rapid and spectacular eruption of phosphorylase activity, far too large to be put down to nucleotide activation and, as the absolute requirement for calcium ions shows, caused by the revival of the phosphorylase kinase. After an interval, depending on the ATP concentration-for this is quickly depleted by contaminating ATPase-the phosphatase regains the upper hand, and the phosphorylase activity vanishes.

In the third paper, Haschke et al. (ibid., 6657) consider the role of phosphorylase phosphatase. They find that magnesium ATP and calcium, the very agencies responsible for unleashing the kinase, also inhibit the phosphatase. The build-up of AMP and the deamination product, IMP, which are both inhibitors of the enzyme, is altogether insufficient to account for the effect. In the absence of evidence to suggest chemical modification it seems likely that there is another inhibiting component in the glycogen particle that regulates it.

\title{
BACTERIOPHAGES
}

Another Enzyme from $\boldsymbol{T}$

by our Molecular Genetics Correspondent

DEFINING completely the functions of the chromosome of a living cell-even one comparatively so simple as a bacteriumis obviously going to take a very long time indeed. But accounting for every function coded by a viral genome may be a more practical proposition. Viruses come in many sizes and shapes, of course, and the trick of success may perhaps be to pick one with the right order of complexity. As lambdologists will ruefully vouch, even a comparatively small virus such as lambda, which has about fifty genes, has a bewildering number of control systems which are proving all too difficult to disentangle.

The very small phages have genomes which are RNA or single stranded DNA;

\section{Evolving Histones?}

IN the past few years histone chemistry has rapidly emerged from the dark ages of not so long ago when histone chemists spent their lives arguing about each others' artefacts. It is true that the function of histones is almost as obscure as ever, but today such is the sophistication of protein chemistry that arguments now centre on the possible evolutionary relationships between histones of known amino-acid sequence rather than on how to fractionate reproducibly various types of histones. Indeed, one type of histone, fraction IV, provides the outstanding example of the evolutionary stability of a protein. Fraction IV histones of pea cell nuclei and calf thymus cell nuclei differ in sequence by only two amino-acids. How long is it since cows and peas diverged from a common ancestor? Such striking evolutionary stability is a characteristic of several types of histone from diverse sources; on the other hand there is evidence that certain histones are species and even tissue specific. Fraction V histones are the best example; these are uniquely restricted to red blood cells, but as Greenaway and Murray speculate in next Wednesday's Nature New Biology, fraction $\mathrm{V}$ histones may be ancestral to the fraction I histones of other cell types.

Greenaway and Murray noticed that the fraction $\mathrm{V}$ histone of chick erythrocytes can be resolved into two fractions, $\mathrm{Va}$ and $\mathrm{Vb}$, by chromatography on 'Amberlite' columns. The two fractions, obtained reproducibly, proved to have the same N-terminal amino-acid, the same electrophoretic mobility and they do not differ in the extent to which they are acetylated, methylated or phosphorylated. In fact, after extensive tests the only difference Greenaway and
Murray found between the two proteins was at the position of the fifteenth aminoacid residue in their sequences. Histone $\mathrm{Va}$ has a glutamine residue at this position whereas histone $\mathrm{Vb}$ has an arginine residue.

These initial experiments were done with histones extracted from the pooled blood from about 1,000 chickens and the obvious question arose as to whether any one bird has both histone $\mathrm{Va}$ and $\mathrm{Vb}$. Experiments with the histones from the blood of individual birds showed that they either had histone $\mathrm{Vb}$ or both $\mathrm{Va}$ and $\mathrm{Vb}$. Furthermore, preliminary analyses of the inheritance of these patterns indicate that in fowl there are probably allelic genes for the two proteins. The existence of chickens homozygous for fraction $\mathrm{Vb}$, however, proves that both alleles are not essential for survival.

The occurrence of such genetic polymorphism among histones increases, of course, the number of distinct histone fractions, but it reduces the complexity of their biological function because such closely related proteins are unlikely to have different functions. Indeed, as Greenaway and Murray suggest, fraction I histones, in cells other than erythrocytes, have so many properties in common with fraction $\mathrm{V}$ histones, restricted to red blood cells, that it is quite feasible that fraction I histones have evolved from fraction $\mathrm{V}$ histones. If this argument has any truth in it, it follows that fraction I histones have rendered the fraction $\mathrm{V}$ histones redundant in all but erythrocytes. The heterogeneity of histone fractions may well belie the range of their functions, which is probably far more restricted than the protein chemist, surveying the numerous fractions from his columns, might be tempted to predict. 